\title{
Demethylation with 5-Aza-2'-deoxycytidine Affects Oxidative Metabolism in Human and Mouse Non-small Cell Lung Cancer Cells
}

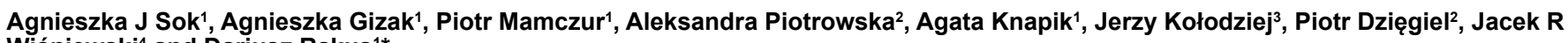
Wiśniewski ${ }^{4}$ and Dariusz Rakus ${ }^{1 *}$

${ }^{1}$ Department of Animal Molecular Physiology, Wroclaw University, Wroclaw, Poland

${ }^{2}$ Department of Histology and Embryology, Wroclaw Medical University, Wroclaw, Poland

${ }^{3}$ Department of Thoracic Surgery, Wroclaw Medical University, Wroclaw, Poland

${ }^{4}$ Department for Proteomics and Signal Transduction, Max-Planck-Institute of Biochemistry, Martinsried, Germany

\begin{abstract}
Background: Non-small cell lung cancer is the most common cause of death from cancer in the world. Here we demonstrate the effect of 5-aza-2'-deoxycytidine (5-dAza), which is one of the most promising anticancer drugs, on the growth and metabolism of mouse (KLN205) and human non-small cell lung cancer-derived (hNSCLC) cells.
\end{abstract}

Results: In both cell lines we observed reduction of the cells viability and rate of their proliferation after 5-dAza treatment. These changes correlated with the lowering of ATP synthesis and lactate release from cells and with overproduction of reactive oxygen species.

5-dAza-treatment of lung cancer cells also induced a decrease of mitochondrial membrane potential and damage of mitochondrial network. These changes were partially reversed by rotenone treatment which also facilitated the cells survival.

Proteomic analysis revealed that the demethylation affected the abundance of proteins associated to energy metabolism, especially those of tricarboxylic acid cycle (TCA) and oxidative phosphorylation metabolism (OXPHOS).

Conclusion: Taken together, our work provide evidence that the mechanism of the toxic effect of 5-dAza basically relies on the alteration of mitochondrial function via dysregulation of proteins involved in TCA and OXPHOS.

Keywords: Decitabine; Fructose-1,6-bisphosphatase; Glycolysis; Mitochondria; Warburg effect

\section{Introduction}

Abnormal methylation of DNA on CpG sequences is one of the most common epigenetic modifications observed in tumors. Hypermethylation of promoter regions of tumor suppressor genes results in their silencing and usually favors tumor progression [1,2]. The cytosine nucleoside analog 5-aza-2'-deoxycytidine (5-dAza, Decitabine) is a widely used demethylating agent [3]. Clinical trials have established the efficacy of 5-dAza in causing disease remission in myelodysplastic syndrome and acute myeloid leukemia [4]. In vitro studies have demonstrated that 5-dAza inhibits growth of several lines of cancer cells, but not fibroblasts [5] or epithelial cells [6]. Nevertheless, the mechanism responsible for the anti-cancer action of this agent remains a matter of controversy.

It has been suggested that 5-dAza may inhibit cancer cells proliferation and/or induce cell death by multiple mechanisms, e.g. by reverting methylation-dependent gene repression of cell cycle inhibitors $\mathrm{p} 21^{\mathrm{WAF}}$ [7] and $\mathrm{p} 15^{\mathrm{INK} 4 \mathrm{~b}}$ [8] and/or up-regulating the expression of caspase-9 [9]. Quite recently, it has been hypothesized that anti-cancer action of 5-dAza, at least in gastric cancer, may be related to the inhibition of glycolysis via renewal of expression of a regulatory gluconeogenic enzyme, fructose-1,6-bisphosphatase 1 (liver FBPase, FBP1, EC 3.1.3.11) [10]. This study suggested that demethylation-induced inhibition of the Warburg effect (conversion of glucose into lactate in the presence of oxygen) together with restoration of the liver FBPase isozyme expression may be a common mechanism of anti-cancer action of 5-dAza. Most recent studies suggested that not only FBP1, but also muscle isozyme of FBPase (FBP2) may affect gastric cancer growth in the manner which is related to the methylation of the enzyme promoter [11].

In line with this hypothesis, it has been demonstrated that promoter-methylation-dependent down-regulation of FBP1 during epithelial-mesenchymal transition of the breast cancer induced glycolysis and increased glucose uptake and production of reactive oxygen species (ROS) [12].

It has been also reported that 5-dAza-induced FBP1 overexpression is related not only to the increase of ROS level but also to the suppression of liver and colon tumor cell lines growth and correlates with induction of $\mathrm{G} 2 / \mathrm{M}$ phase cell cycle arrest [13].

In the present paper, we show that the global demethylation with 5-dAza affects the expression of mRNA for FBPase isozymes as well as for the regulatory enzymes of glycolysis in the explant-derived human non-small cells lung cancer (the hNSCLC cells) and mouse squamous cell lung cancer (the KLN205 cell line). However, we found no evidence

*Corresponding author: Dariusz Rakus, Department of Animal Molecular Physiology, Wroclaw University, Cybulskiego 30, 50-205 Wroclaw, Poland, Tel/ Fax: +48 71 3759213; E-mail: drakus@biol.uni.wroc.pl

Received December 19, 2013; Accepted February 17, 2014; Published February 20, 2014

Citation: Sok AJ, Gizak A, Mamczur P, Piotrowska A, Knapik A, et al. (2014) Demethylation with 5-Aza-2'-deoxycytidine Affects Oxidative Metabolism in Human and Mouse Non-small Cell Lung Cancer Cells. J Cancer Sci Ther 6: 036-044. doi:10.4172/1948-5956.1000246

Copyright: (c) 2014 Sok AJ, et al. This is an open-access article distributed under the terms of the Creative Commons Attribution License, which permits unrestricted use, distribution, and reproduction in any medium, provided the original author and source are credited. 
that these changes might modulate the Warburg effect in the lung cancer cells.

Based on results of our proteomic and metabolic studies we hypothesize that the mechanism of the toxic effect of 5-dAza and the apparent inhibition of glycolysis by this compound is a result of an alteration of energetic metabolism of mitochondria.

\section{Material and Methods}

\section{Chemicals}

Unless stated otherwise, all chemicals were from Sigma-Aldrich (St. Louis, USA) and were of the cell culture grade.

\section{Cell culture}

All cell lines were cultured at $37^{\circ} \mathrm{C}$ in a humidified atmosphere with $5 \% \mathrm{CO}_{2}$ and maintained using standard cell culture techniques. The KLN205 cells (ATCC ${ }^{\circledR}$ CRL-1453 ${ }^{\mathrm{TM}}$ ) were cultured in Eagle's Minimum Essential Medium. The primary culture of explant-derived human lung cells (hNSCLC) was prepared according to previously established methods [14]. Immediately after surgery the histologically proven Non-Small Cell Lung Cancer tumor fragments were put into Hank's Balanced Salt solution, dissected with scissor into about $1 \mathrm{~mm}^{3}$ sections, and put into culture dishes coated with Matrigel. The primary cultures of hNSCLC cells were cultured in Dulbecco's Modified Eagle Medium (DMEM). To avoid fibroblasts outgrowth of cancer primary cultures, L-valine was substituted to D-valine (0.094 $\mathrm{g} / \mathrm{L}$ ) [15]. In order to verify the purity of explant-derived hNSCLC cell line, the immunostaining for cytokeratin-7, a marker of cancer cells was performed (data not shown) [16].

Both the culture media, for the KLN205 and for the hNSCLC, were supplemented with $2 \mathrm{mM}$ glutamine, $1 \%$ non-essential amino acids, penicillin $(100 \mathrm{U} / \mathrm{mL})$, streptomycin $(0.1 \mathrm{mg} / \mathrm{mL})$ and $10 \%$ fetal bovine serum (FBS). The cells were seeded in the initial density $3 \times 10^{3}$ cells $/ \mathrm{cm}^{2}$ and cultured for 24-192 hours depending on experimental procedure.

Prior to the treatment with 5-dAza and/or rotenone the cells were cultured at least for $24 \mathrm{~h}$ under standard conditions.

In experiments with 5-dAza, the medium containing freshly prepared $5 \mu \mathrm{M} 5$-dAza (in acidic water, $\mathrm{pH} 4$ ) was added to the cell culture and exchanged every $24 \mathrm{~h}$. In the controls, the equal volume of acidic water was added instead of $5 \mu \mathrm{M} 5$-dAza.

In experiments with rotenone, the cells were incubated with $1 \mu \mathrm{M}$ rotenone dissolved in DMSO or with appropriate volume of DMSO for control culture. When both agents were used together, rotenone was added 15 minutes before demethylation procedure.

In the experiments, the concentration of DMSO in culture media did not exceed $0.02 \%$.

\section{Genomic DNA isolation and analysis of global methylation}

Genomic DNA was purified from the control and 5-dAza treated the hNSCLC cells using GeneJETTM Genomic DNA Purification Kit (Fermentas) according to the manufacturer's protocol. Demethylation was carried out for $72 \mathrm{~h}$ before DNA isolation. To measure global DNA methylation shifts after 5-dAza treatment genomic DNA was tested with Imprint ${ }^{\circledR}$ Methylated DNA Quantification Kit according to the manufacturer's instructions.

\section{Analysis of Gene Expression}

Total RNA from cell cultures was obtained by phenol/chloroform extraction with TRI Reagent (Applied Biosystems). First strand cDNA was synthesized from the total RNA using the High-Capacity cDNA Reverse Transcription Kit (Applied Biosystems) according to the manufacturer's protocol. Real-time PCR was performed with commercially available TaqMan probes (Applied Biosystems) for the hNSCLC cells using PCR Master Mix (Applied Biosystems) and with gene-specific primers for the KLN205 cells using SYBR ${ }^{\circledR}$ Green PCR Master Mix (Applied Biosystems). Amplification primers and catalog numbers of TaqMan probes are listed in Table 1. Quantities of each transcript were normalized to HPRT1 gene for the hNSCLC cells and to RNA polymerase II alpha gene for the KLN205 cells as an internal control. A negative control lacking cDNA was included in each assay. Amplification was performed for each cDNA samples in triplicate and the relative expression ratio was calculated according to the Pfaffl method [17].

\section{Immunochemistry}

The hNSCLC and the KLN205 cells were fixed in $4 \%$ paraformaldehyde for $15 \mathrm{~min}$ at room temperature (RT), washed with PBS, permeabilized with $0.3 \%$ Triton X-100/PBS and used for immunocytochemical staining. To avoid the unspecific binding of immunoglobulins, the cells were incubated with 3\% BSA in PBS for $1 \mathrm{~h}$ at RT before the application of primary antibodies.

To determine the subcellular localization of cell proliferation marker (Ki67), the cells were incubated overnight at $4^{\circ} \mathrm{C}$ with rabbit polyclonal anti-Ki67 antibodies (1:1000) and subsequently with fluorophore-labeled secondary antibodies (goat anti-rabbit-FITC; 1:2000) diluted in PBS containing 0.5\% BSA. The excess of antibodies was removed by washings with $0.1 \%$ Triton X-100/PBS. In control reactions, the primary antibodies were omitted. To visualize the nuclei, the cells were counterstained with DAPI.

The localization of proteins was examined with Olympus IX71 fluorescence microscope equipped with Cell^F software (Olympus Soft Imaging Solutions GmbH) and/or Olympus FV1000 confocal microscope.

Ki67 index was calculated as the fraction of Ki67-positive tumor cells. Mitotic index was calculated as the ratio between the number of cells in mitosis and the total number of cells.

\section{Measurement of cell viability}

Cells were seeded in 12-well plates at density of 1000 cells per $\mathrm{cm}^{2}$ for the KLN205 and of 6000 cells per $\mathrm{cm}^{2}$ for the hNSCLC and kept under standard conditions for $24 \mathrm{~h}$ and $96 \mathrm{~h}$, respectively. Then, the cells were washed with HBSS and treated with $5 \mu \mathrm{M} 5$-dAza. The medium containing 5-dAza was exchanged every $24 \mathrm{~h}$.

MTT assay was performed starting on 24 hours after adding 5-dAza to the cells and continued every 24 hours for 8 days. MTT stock of 10 $\mathrm{mg} / \mathrm{mL}$ in distilled water was always freshly prepared. The cells were rinsed twice with sterilized PBS and the solution of $1 \mathrm{mg} / \mathrm{mL}$ MTT in RPMI medium (local vendor) without serum was added to cell cultures. After $3 \mathrm{~h}$ of incubation in standard conditions cells were washed with PBS and MTT crystals have been dissolved in DMSO at $37^{\circ} \mathrm{C}$ in the dark. The absorbance was measured at $560 \mathrm{~nm}$ with a reference filter of $670 \mathrm{~nm}$ using a plate reader ASYS UVM340 (Biogenet). All measurements were taken in triplicate. The number of viable cells was 


\section{Gene name phosphofructokinase liver (PFKL) pyruvate kinase muscle 1 (PKM1) pyruvate kinase muscle 2 (PKM2) tumor protein 53 (TP53) nuclear factor $\mathrm{KB}$ (NFKB1) hypoxia inducible factor $1 \mathrm{~A}$ (HIF1A)}

fructose-1,6-bisphosphatase 1 (FBP1) foward- CAGCTGCTGAATTCGCTCTG reversed- ACATTGGTTGAGCCAGCGATA fructose-1,6-bisphosphatase 2 (FBP2) foward- GACCCTCTGGATGGATCTTCA reversed- CAGAAGGCTCATCCTCCGT phosphofructokinase platelet (PFKP) foward- CTATGACGGCTTTGAAGGATTC reversed- GACATCTGCCCAGCCGATT phosphofructokinase muscle (PFKM) foward- CAACCGGGTGCTGGTCGTA reversed- CTCCAGCCAGCCTCCTCA foward- GTGCGAGCCTCCAGTCACT reversed- CGGTACCTGGCCACTTGGT foward- ACTACCCTCTGGAGGCTGTT reversed- GCGGCGGAGTTCCTCGAAT foward- TTGGGACCATCCTGGCTGTA reversed- TGACTCCTCCATGGCAGTCA foward- GACCTTGCCTATCTACAAGCA reversed- CATCTCCTTGGTCTGCTGCA foward- CAGCCAGCAAGTCCTTCTGA reversed- GCTGGGAAAAGTTAGGAGTGT foward- GGCTGGCAAGGCCATTGGA reversed- GAAGACTTTGGCCCCCACATA
TaqMan probes (Assay ID) for hNSCLC

Hs00166829_m1

Hs00427791_m1

Hs00242993_m1

Hs00175997_m1

Hs00160027 m1

Hs00987255_m1

Hs00987261_g1

Hs01034249 m1

Hs00765730_m1

Hs00153153 m1

Table 1: Primers and probes used in qPCR experiments.

calculated as a percentage of the value obtained from the control cells incubated in standard culture media.

\section{Lactate assay}

Lactate assay kit, based on spectrophotometric measurement of $\mathrm{NAD}^{+}$reduction at $340 \mathrm{~nm}$ was used to check the effect of 5-dAza on lactate release into culture medium. The samples of culture media from the KLN205 and NSCLC cells treated for $48 \mathrm{~h}$ with 5 -dAza were precipitated with $8 \%$ perchloric acid and centrifuged at $14,000 \mathrm{xg}$ for $5 \mathrm{~min}$. at $4^{\circ} \mathrm{C}$. The supernatants were neutralized with 2 $\mathrm{M} \mathrm{KOH}$, centrifuged again and used for the measurements of lactate concentration. Standard reaction mixture contained: $0.6 \mathrm{M}$ glycine, 0.2 $\mathrm{M}$ hydrazine sulphate, $2.6 \mathrm{mM}$ EDTA, $2.5 \mathrm{mM} \mathrm{NAD}^{+}, \mathrm{pH} 9.5,37^{\circ} \mathrm{C}$ and $50 \mu$ of the supernatant [18]. The reaction was started by addition of $1 \mathrm{U}$ of lactate dehydrogenase. The measurement of $\mathrm{NAD}^{+}$reduction monitored at $340 \mathrm{~nm}$ was performed with the Agilent 8452A diode array spectrophotometer.

\section{ATP assay}

ATP measurements were performed in Infinite ${ }^{\circledR} 200$ PRO (Tecan) multimode reader using ATPlite ${ }^{\mathrm{TM}}$ (PerkinElmer) assay based on the luciferase-luciferin system. KLN205 cells were cultured in 96-well white plates (GreinerBioOne) at density of 1000 cells per $\mathrm{cm}^{2}$. After 24 $\mathrm{h}$ of culture under standard conditions cells were incubated for $24 \mathrm{~h}$ and $48 \mathrm{~h}$ with $5 \mu \mathrm{M} 5$-dAza and/or $1 \mu \mathrm{M}$ rotenone. The medium containing 5 -dAza and rotenone was exchanged every $24 \mathrm{~h}$. To generate standard curve for calculation of ATP concentration in samples and controls, defined amounts of ATP (provided with kit) were added to the KLN205 cells culture medium. Samples and ATP standard curve were done at least in triplicate.

\section{Measurement of reactive oxygen species (ROS)}

Intracellular ROS production was detected using a fluorescent ROS-sensitive probe, MitoTracker ${ }^{\circledR}$ red CM-H XRos (Molecular Probes). hNSCLC cells were treated with 5-dAza, rotenone, or both the compounds together as described above. After $24 \mathrm{~h}$ the cells were loaded with the fluorescent probe $\left(0.2 \mu \mathrm{M}, 30 \mathrm{~min}, 37^{\circ} \mathrm{C}\right)$, fixed in paraformaldehyde and examined with confocal microscopy. The quantitative measurements of the fluorescence signal was performed using Cell^F software (Olympus Soft Imaging Solutions GmbH).

\section{Mitochondrial membrane potential}

The hNSCLC cells growing on coverslips were incubated for $24 \mathrm{~h}$ with $5 \mu \mathrm{M} 5$-dAza or $1 \mu \mathrm{M}$ rotenone or the two compounds together and then stained with the JC-1 fluorescent dye (Mitochondrial Permeability Transition Detection Kit, AbD Serotec). The color of the dye changes reversibly from green to red. Polarized mitochondria accumulate more of the dye and are red. In cells with depolarized mitochondria, most of the dye is dispersed throughout cytoplasm and fluoresce green. The hNSCLC cells were incubated with JC- 1 for $30 \mathrm{~min}$ at $37^{\circ} \mathrm{C}$ and the fluorescence was observed in confocal microscope. During the observations, to prevent fluorescence changes resulting from redistribution of the dye, cells remained in the JC-1 bath. Excitation of the dye was at $488 \mathrm{~nm}$ and the emission was observed using a long pass filter, which allowed for simultaneous observation of green (monomers of JC1) and red (aggregates of the dye) fluorescence.

\section{Proteomic analysis}

Cell pellets were dissolved in 2\% SDS, $100 \mathrm{mM} \mathrm{DTT,} 0.1 \mathrm{M}$ Tris- $\mathrm{HCl}, \mathrm{pH} 7.6$ at $100^{\circ} \mathrm{C}$ for $5 \mathrm{~min}$. Aliquots containing $50 \mu \mathrm{g}$ of total protein were processed in the $30 \mathrm{k}$ filtration units (Millipore, Cat No. MRCF0R030) according to the MED-FASP protocol using endoproteinase Lys-C and trypsin [19,20]. The resulting Lys-C and tryptic peptides were separated into 4 and 2 SAX fractions, respectively [21] and were analyzed using Orbitrap instrument as described previously [20]. Samples were processed and analyzed in triplicates.

The MS data were analyzed using the software environment of MaxQuant version 1.2.6.20 [22]. Proteins were identified by searching MS and MS/MS data of peptides against a decoy version of the UniProtKB/Swiss-Prot. Carbamidomethylation of cysteines was set as fixed modification. The maximum false peptide discovery rate was specified as 0.01 . Absolute protein contents were calculated by the TPA approach [23] using LFQ intensity values [24]. Statistical analysis of the data was performed as described previously [23].

\section{Results}

\section{5-dAza reduces methylation state of DNA in cancer cells}

The analysis of the genomic DNA isolated from the hNSCLC cells cultured for 72 hours with $5 \mu \mathrm{M} 5$-dAza revealed that these cells displayed 30\% ( $\mathrm{SD}=7$ ) lower methylation state of DNA than the control, 5-dAza-untreated, cells. The results of the experiment confirmed that the application of $5 \mu \mathrm{M} 5$-dAza may be used to study the effect of DNA demethylation on cancer cells biology.

\section{5-dAza triggers reduction of lung cancer cells growth}

The treatment of cells with $5 \mu \mathrm{M} 5$-dAza reduced the rate of proliferation of both the KLN205 and the hNSCLC cells. 72hour incubation with 5-dAza substantially lowered the rate of cell proliferation as measured by the MMT assay. This decrease correlated with a significantly lower level of the Ki67 staining and mitotic index (Figure 1). The mitotic index decreased from 0.45 and 0.52 , respectively 
Citation: Sok AJ, Gizak A, Mamczur P, Piotrowska A, Knapik A, et al. (2014) Demethylation with 5-Aza-2'-deoxycytidine Affects Oxidative Metabolism in Human and Mouse Non-small Cell Lung Cancer Cells. J Cancer Sci Ther 6: 036-044. doi:10.4172/1948-5956.1000246

for the hNSCLC and the KLN205, to zero in both the cell lines. The Ki67 index was reduced by approximately $32-35 \%$ in both the cell lines.

After 8 days of 5-dAza treatment the amount of the KLN205 cells was about 2.5-times lower than the amount of the control cells (Figure 1, bottom right). Similar decrease was observed in the case of the hNSCLC cells (Figure 1, top right).

\section{Demethylation influences the level of mRNA for FBPase, glycolytic enzymes and TP53}

The expression of selected genes coding the isozymes of regulatory enzymes of carbohydrate metabolism (isozymes of - PFK: phosphofructokinase, PKM: muscle isoforms of pyruvate kinase and FBPase) and for proteins crucial for cancer metabolism (TP53: tumor protein 53, NFKB1: nuclear factor of kappa light polypeptide gene enhancer in B-cells 1 and HIF1A: hypoxia inducible factor 1) were quantified by RT-qPCR in 5-dAza treated and non-treated hNSCLC and KLN205 cells.

The mRNAs for glycolytic enzymes were slightly elevated in 5-dAza-treated cells although the changes were not statistically significant, except mRNA for PKM1 in the hNSCLC cells (Figure 2A). The most statistically significant changes concerned the mRNA for FBPase isozymes (Figures 2A and 2B) and TP53 (Figure 2A), whereas practically no changes were observed in the mRNA level for HIF1A and NFKB1 (data not shown).

Although demethylation caused 2- to 4-fold increase of the mRNA level for FBPases, the amount of the mRNA for both FBPase isozymes was at least 10 times lower than the amount of mRNA for glycolytic enzymes (data not shown) suggesting that gluco- and glyconeogenesis is not able to significantly affect the flux through glycolysis.

\section{Demethylation affects the amount of metabolic proteins as measured by quantitative mass spectrometry}

To provide a deeper insight in the effect of the drug on proteins abundance we have analyzed the whole cell lysates from the hNSCLC cells using mass spectrometry techniques. The 5-dAza-treated cells and the untreated controls were analyzed in triplicate using the MED-FASP approach. The entire analysis allowed identification of 6,400 proteins. 4,750 proteins which were identified at least 2 times in one of the samples were used for label-free quantitative analysis. The most significant changes in the 5-dAza-treated hNSCLC cells were observed for seven proteins $\left(\mathrm{p}<10^{-4}\right)$ : Phosphoenolpyruvate carboxykinase (PEPCK), Aldehyde dehydrogenase family 16 member, Tripeptidyl peptidase 1 , Collagen alpha-1 (XII) chain, tRNA-guanine transglycosylase, ATP synthase subunit beta and Tyrosine-protein kinase-like 7 .

The analysis also revealed that the total abundances of proteins involved in glycolysis and gluconeogenesis (Gly/Glu), tricarboxylic acid cycle (TCA), pentose phosphate pathway (PPP), and oxidative phosphorylation (OXPHOS) rose slightly after the demethylation (Figure 3).

Glycolysis and gluconeogenesis: 5-dAza treatment of cancer cells did not lowered, but in most cases slightly elevated, the abundance of glycolytic enzymes and glucose and lactate transporters in the hNSCLC cells (Figure 4). The only significant exception was enolase 2 . However, this isozyme consists of only $4.1 \%$ of the total enolase and it should not significantly affect the glycolytic flux. This suggests that the decrease in release of lactate from 5-dAza-treated cells is not a result of downregulation of glycolytic pathway but is an epiphenomenon.
The most significant increase concerned phosphoenolpyruvate carboxykinase (PEPCK) which is one of the regulatory enzymes of gluconeogenesis. This elevation did not correlate with the presence of

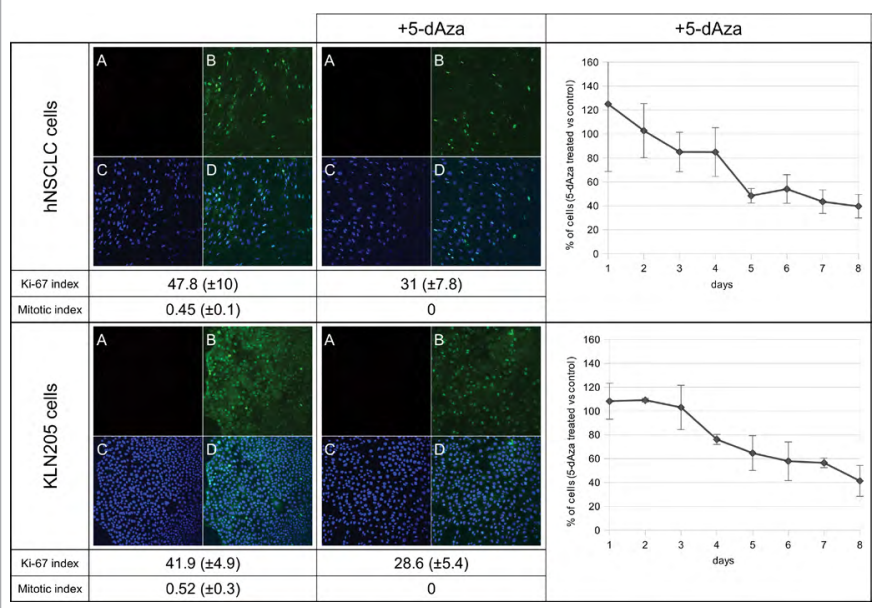

Figure 1: The effect of 5-dAza treatment on the rate of proliferation of the hNSCLC and the KLN205 cells. Left panel shows the changes in Ki67 staining: A) the control staining, B) Ki67 staining, C) DAPI staining, D) merged pictures $B$ and $C$. Right panel demonstrates viability of cells culturing in the presence of 5-dAza. Plots present mean results of 3 independent experiments calculated as a percentage of treated versus non-treated cells.
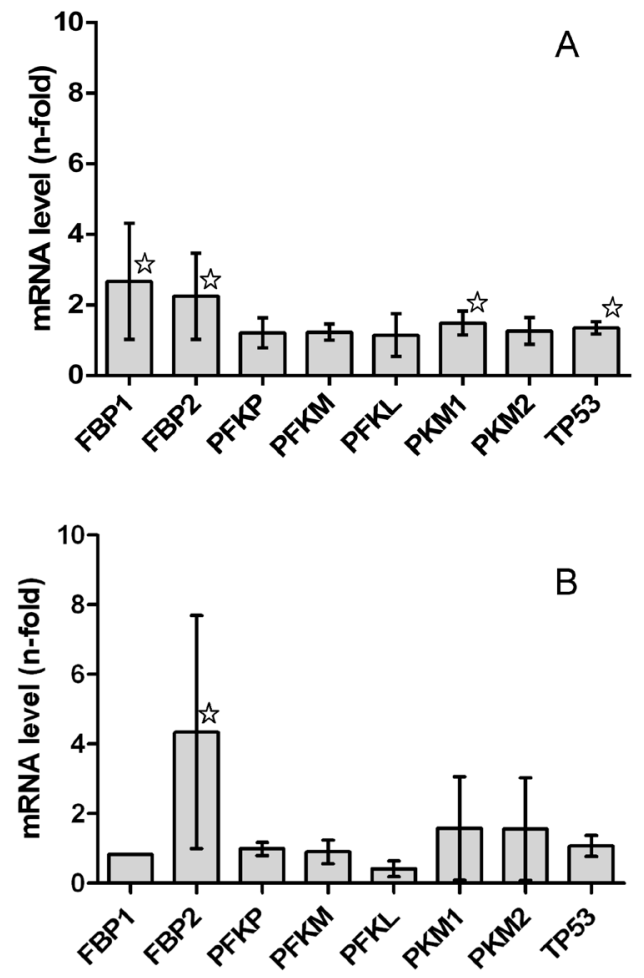

Figure 2: Gene expression changes in cancer cell lines after demethylation. The $y$-axis represents the relative fold difference in mRNA level between $5-\mathrm{dAza}$ treated cells and control and was calculated using the $2^{-\Delta \Delta \mathrm{Ct}}$ formula (Paffl, 2001). A) Mean \pm SD of 4 experiments for the hNSCLC cells and B) mean \pm SD of 3 experiments for the KLN205 cells; asterisk indicates that p $<0.05$ vs. control. 


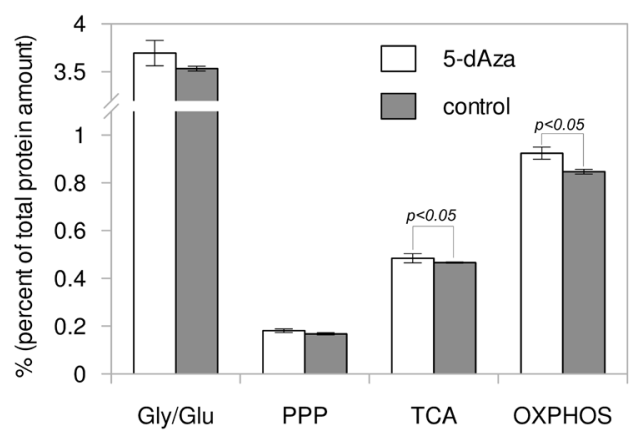

Figure 3: Relative changes in the abundances of proteins involved in carbohydrate metabolism and energy production in the hNSCLC cells after 5-dAza treatment. Total abundances of proteins involved in glycolysis and gluconeogenesis (Gly/Glu), tricarboxylic acid cycle (TCA), pentose phosphate pathway (PPP), and oxidative phosphorylation (OXPHOS) were calculated by summarizing total protein content in individual samples. The relative abundances for individual categories were calculated by dividing these values by the total amount of proteins in the sample. Plot shows a mean \pm SD of 3 measurements.

substantial amount of other regulatory enzymes of glycogen/glucose synthesis from non-carbohydrates. We were not able to detect pyruvate carboxylase and FBPase which indicates that the amount of both the enzymes was at least one hundred times lower than that of PEPCK and about 100-10,000 lower than the abundance of glycolytic enzymes.

Pentose phosphate pathway: Demethylation did not change or slightly increased the amount of practically all enzymes of PPP (Figure 4). The most pronounced changes concerned 6-phosphogluconolactonase, which amount was elevated nearly two-fold. However, the enzyme is not considered to be a regulatory point of the PPP.

Pyruvate dehydrogenase complex, glutaminase and tricarboxylic acid cycle: Pyruvate dehydrogenase complex is the main point of energetic substrates delivery to TCA. Our studies demonstrated that the demethylation increased significantly the amout of dihydrolipoamide dehydrogenase (Figure 4). A similar trend - an increase of the amount of proteins, was observed also for the TCA enzymes and for the enzymes supporting this pathway with $\alpha$-ketoglutarate (glutaminase and glutamate oxaloacetate transaminase).

Oxidative phosphorylation: In contrast to the foregoing pathways and processes, the changes concerning OXPHOS complexes were not uniform. Except for the complex II (succinate dehydrogenase), the treatment with 5-dAza disturbed the stoichiometric ratios of proteins within OXPHOS complexes and the most significant changes were related to the Complex I and III (Figure 4).

\section{Demethylation reduces lactate production}

Previously, it has been shown that 5-dAza inhibits lactate production in gastric cancer cells [10]. To check whether the decrease in lactate production after the global demethylation is a common mechanism of 5-dAza action we measured the concentration of lactate in media in which the lung cancer cells were cultured. Figure 5 shows that 5-dAza treatment of lung cancer cells decreased the lactate release from the KLN205 and the hNSCLC cells by $16 \%$ and 30\%, respectively.

\section{5-dAza dysregulates mitochondrial function}

24-hour demethylation of the hNSCLC cells resulted in the decrease of ATP production (Figure 6A) and in the increase of reactive oxygen species (ROS) (Figure 6B) which correlated with dramatic reduction of mitochondria membrane polarization and in fragmentation of their network (Figures 7A and 7B)

\section{Rotenone antagonizes the 5-dAza effect on the mitochondrial membrane polarization, ROS production and rate of cell proliferation but not on the ATP synthesis}

We found that rotenone treatment of lung cancer cells resulted in the significant increase of ROS production (Figure 6B), decrease of ATP production (Figure 6A) and loss of mitochondria membrane polarization (Figure 7), similarly to treatment of the cells with 5-dAza. As an effect, the lowered viability of cells was observed (Figure 8).

Rotenone enhances mitochondrial ROS production by blocking the transfer of electrons from iron-sulfur centers in Complex I to ubiquinone [25]. However, it was also shown that if Complex III becomes the main system of ROS production in a cell, blockade of the electron flow to this complex by rotenone results in decrease of ROS production and reduction of mitochondria damage and that rotenone prevents ROS generation induced by antimycin A - a known inhibitor of Complex III, in mitochondria oxidizing Complex I substrates [26].

Since 5-dAza not only stimulated ROS production but also disturbed the stoichiometric ratios of proteins within OXPHOS complexes, we attempted to check which of the complexes is responsible for this 5-dAza-stimulated formation of ROS.

The result presented here revealed that simultaneous exposition of the lung cancer cells to 5-dAza and rotenone considerably restored the 5-dAza-evoked and rotenone-evoked increase of ROS, loss of mitochondrial membrane polarity and their network integrity (Figure 7). However, the treatment of lung cancer cells with both the compounds together did not restored significantly ATP level (Figure 6A). This suggests that 5-dAza induces formation of ROS by mitochondrial Complex III.

The treatment of hNSCLC cells with 5-dAza or rotenone resulted in similar decrease in the rate of proliferation for about $20 \%$ after 2 days of the treatment and $60 \%$ after 4 days (Figure 8 ). The simultaneous treatment of the cells with both the compounds substantially increased the rate of cell proliferation (Figure 8).

\section{Discussion}

A growing body of evidence indicates that chemical demethylation of tumor suppressor genes promoters may be a useful strategy for anticancer therapy. One of the most promising agents used in therapy and clinical trials for selected malignancies, e.g. myelodysplastic syndrome [27], acute myeloid leukemia [28] and non-small-cell lung cancer $[29,30]$ is 5-Aza-2'-deoxycytidine. 5-dAza is one of the DNA methyltransferase (DNMT) inhibitors, the family of nucleoside analogs which may be incorporated into newly synthesized DNA resulting in covalent trapping of DNMT. 5-dAza may also mediate DNA damage by leading to formation of DNA double-strand breaks [31].

Several genes, mostly these involved in the regulation of cell cycle and induction of apoptosis, have been proposed to be targets of 5-dAza actions [32-36]. Latest studies have suggested that 5-dAza may inhibit the growth of gastric, liver and colon cancer cell lines elevating the level of the FBPase isozymes $[10,11,13]$. It has been shown that in these cancers, FBP1 and FBP2 promoters are hypermethylated $[11,13]$ and that pharmacological demethylation restores the expression of FBP1 which is correlated with the G2/M phase cell cycle arrest [13] and with 
Citation: Sok AJ, Gizak A, Mamczur P, Piotrowska A, Knapik A, et al. (2014) Demethylation with 5-Aza-2'-deoxycytidine Affects Oxidative Metabolism in Human and Mouse Non-small Cell Lung Cancer Cells. J Cancer Sci Ther 6: 036-044. doi:10.4172/1948-5956.1000246

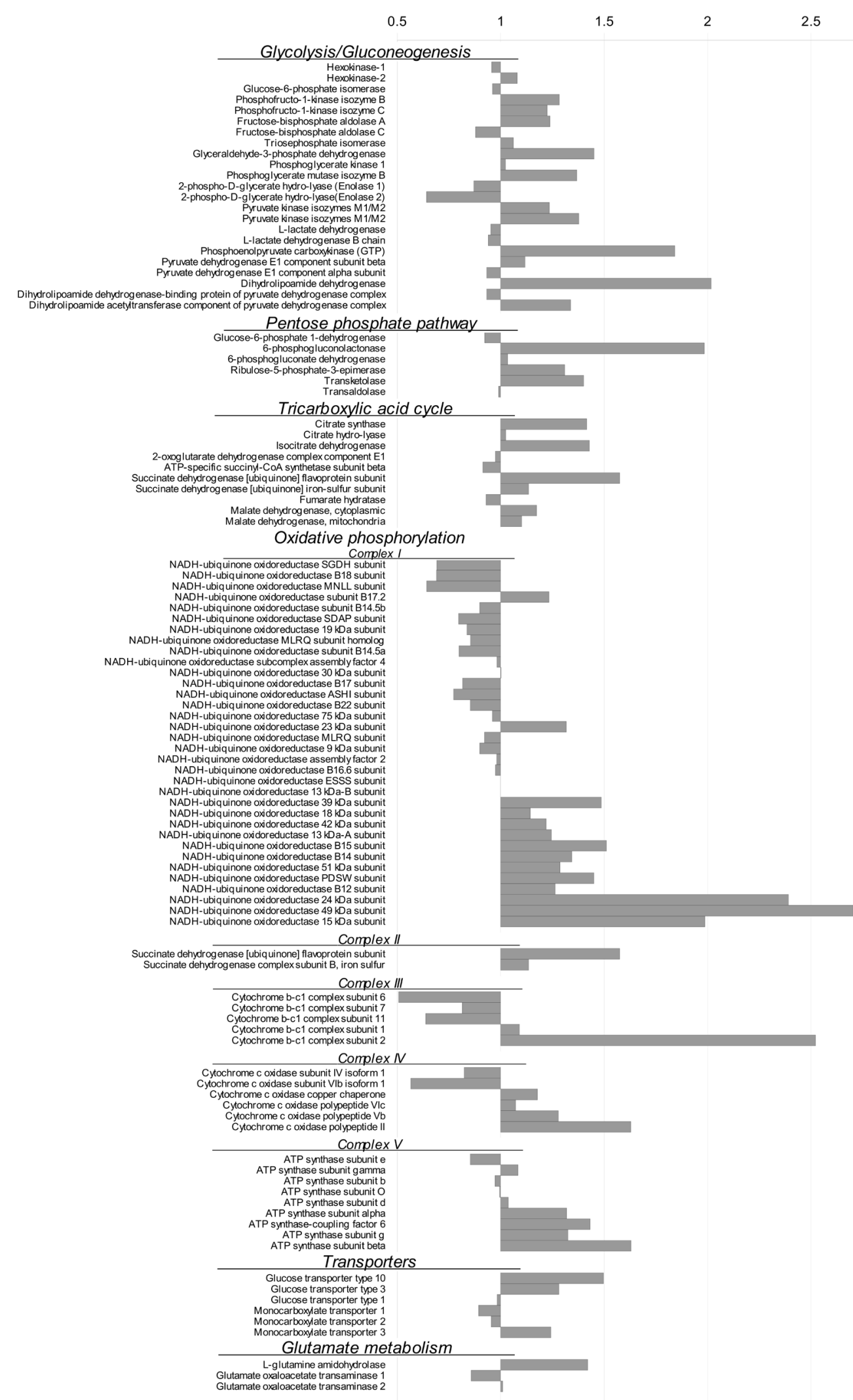

Figure 4: Changes in the abundance of metabolic enzymes after 5-dAza treatment of the hNSCLC cells. Metabolic enzymes identified in quantitative mass spectrometry were classified to the one of ten group of metabolic protein. Grey bars present ratio of amount of protein detected in 5-dAza treated cells to control cells. 


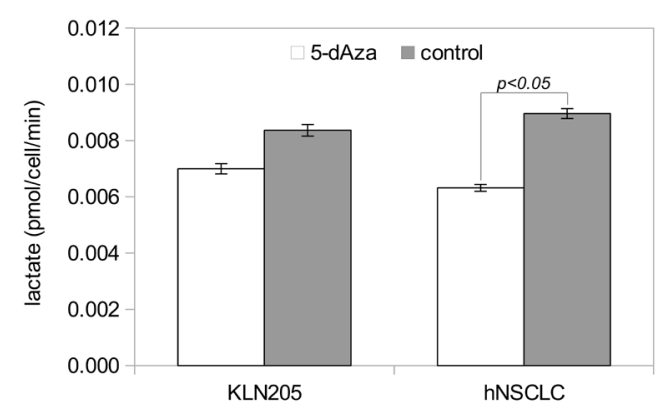

Figure 5: Lactate release from the cancer cells after demethylation with 5-dAza. The concentration of lactate released into the culture medium after 2-days treatment with 5-dAza was significantly lower (empty boxes) than in the control (shaded boxes).
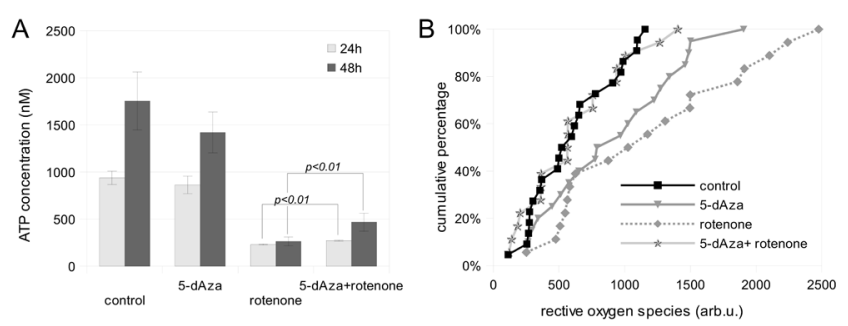

Figure 6: Effect of 5-dAza and rotenone on ATP level and ROS production in lung cancer cell line. A) Treatment of cells with rotenone dramatically decreased the ATP level. Application of 5-dAza and rotenone together only slightly restored ATP production. Concentration of ATP in each sample after 24 and 48 hours of 5-dAza and/or rotenone treatment was calculated using standard curve. Bars represent the mean of three experiments \pm SD and asterisk indicates that $p<0.05$ vs. control; B) Cumulative percentage of ROS production after 24 hours cells culture with 5-dAza and/or rotenone. Shift of the curves to the right ( $\nabla-5-d A z a$ and - -rotenone) relatively to the control curve indicates increase in ROS production.

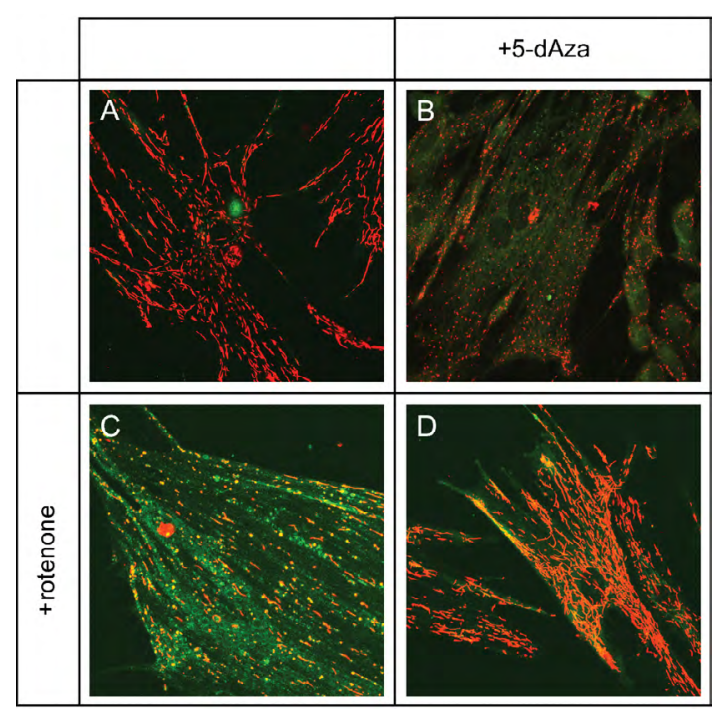

Figure 7: The effect of 5-dAza and rotenone on mitochondria membrane polarization and their network in the hNSCLC. Panel A shows polarization of mitochondrial network of control cells cultured in standard conditions. 24-hours treatment of the cells with $5 \mu \mathrm{M} 5$-dAza (B) and $1 \mu \mathrm{M}$ rotenone (C) reduced mitochondrial membrane polarization and disrupted mitochondrial network. A simultaneous application of both the agents (D) reversed the toxic effect.

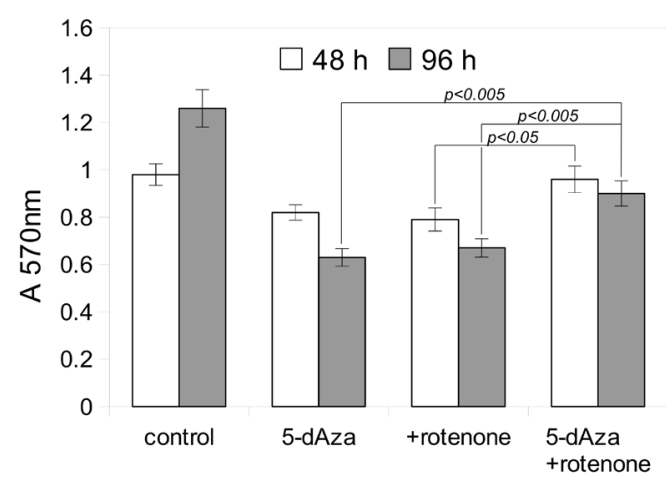

Figure 8: Viability of the hNSCLC cells after 5-dAza and rotenone treatment. Viability was measured using the MTT assay after 48 and 96 hours of the cell culture exposition to $5 \mu \mathrm{M}$ 5-dAza and/or $1 \mu \mathrm{M}$ rotenone. 5-dAza and rotenone alone reduced significantly $(p<0.05)$ the rate of proliferation of the lung cancer cells whereas the simultaneous application of both the compounds partially abrogated the toxic effect. Bars represent the mean of three experiments \pm SE.

the inhibition of lactate production [10]. On the basis of these findings it has been proposed that FBP1 might function as a tumor suppressor protein and the up-regulation of FBP1 may be a mechanism of the Warburg effect suppression.

\section{The lack of evidence for glycolysis-related effect of 5-dAza on lung cancer cell viability}

The manner in which FBPase could hypothetically inhibit the glycolytic flux and the Warburg effect is related to the existence of a substrate cycle phenomenon which may be theoretically formed between FBPase and PFK. PFK catalysing phosphorylation of fructose6-phosphate to fructose-1,6-bisphosphate - the reaction reverse to that catalysed by FBPase, is a regulatory enzyme of glycolysis. The substrate cycle may occur when both the opposing reactions catalysed by different enzymes act simultaneously. The result of the cycle is lowering of net substrate-to-product conversion and, as it is in the case of the cycle formed by FBPase and PFK, the depletion of ATP level (in such case, the substrate cycle is called the futile cycle). The basic requirement for the substrate cycle is that the activities of both enzymes forming the cycle are similar. Since the specific activity of mammalian FBPase is much lower than mammalian PFK [37] thus, to ensure the efficient functioning of the cycle, the concentration of FBPase must be higher than PFK.

Our studies revealed that the level of mRNA for both FBPase isozymes, even after demethylation, was at least two orders of magnitude lower than the abundance of mRNA for PFK isozymes. Moreover, the quantitative proteomic analysis and the measurement of the enzyme activity (data not shown) demonstrated that the amount of FBPase protein in the lung cancer cell lines was below of the limit of detection. From this, it is clear that in lung cancers, FBPase is not able to form an efficient substrate cycle and hence, it cannot affect the flux through glycolysis and the release of lactate nor modulate the Warburg effect.

Because our results revealed that 5-dAza significantly reduced the rate of lactate production we checked whether the compound decreased the abundance of glycolytic enzymes. Unexpectedly, we found that the 5-dAza treatment of cancer cells increased the amount of mRNAs for the regulatory enzymes of glycolysis: PFK (for its all three isozymes: the 
liver, muscle and platelet) and pyruvate kinase (both for PKM1 - the muscle type, and PKM2 - the embryonic one). The mRNAs level for other glycolytic enzymes were not lowered indicating that glycolytic capacity of cells treated with 5-dAza was not diminished.

The quantitative proteomic analysis confirmed that 5-dAza did not lowered, but in most cases elevated, the abundance of glycolytic enzymes in the hNSCLC cells. This indicates that the decrease in release of lactate from 5-dAza-treated cells is not a result of down-regulation of glycolytic pathway but is an epiphenomenon.

\section{5-dAza dysregulates mitochondrial metabolism}

One of the explanation of the decreased lactate release after 5 -dAza treatment might be an increase in oxidation of pyruvate by mitochondria. Indeed, we found that 5-dAza elevated the titer of most of the TCA enzymes (Figure 4) and significantly increased the amount of dihydrolipoamide dehydrogenase which is the crucial component of pyruvate dehydrogenase complex.

Studying the 5-dAza-induced changes in mitochondrial proteome we found that the compound dysregulated the expression of components of OXPHOS complexes (Figure 4) reduced the ATP synthesis and elevated the ROS production which was correlated with the loss of mitochondrial membrane potential and the damage of mitochondrial network.

Thus, it might be hypothesized that the toxic effect of 5-dAza is mainly related to the overproduction of ROS by hyperactive and dysregulated mitochondria. To verify this, we suppressed mitochondrial respiration with rotenone. This compound is known to stimulate ROS production. However, in mitochondria oxidizing Complex I substrates (such as pyruvate), if the main system of ROS production is Complex III rotenone blocks ROS generation induced by inhibitors of this complex [26]. When we stimulated lung cancer cells simultaneously with rotenone and 5-dAza we observed the significant reversal of the 5-dAza-evoked decrease of mitochondrial membranes polarization and mortality of cancer cells with no effect on the 5-dAzaor rotenone-induced decrease of ATP level. The foregoing results point to Complex III as the main free radicals generator after 5-dAza treatment in lung cancer cells. They also suggest that the elevation of ROS production is the primeval cause of lung cancer cells mortality after 5-dAza treatment.

\section{Concluding Remarks}

The global demethylation of cells with 5-dAza affects the expression of several genes containing the CpG islands within their promoters but the extension and direction of the changes depends on a cellular context, e.g. on the basal level of $\mathrm{CpG}$ islands methylation and on the activity of histone deacylase etc. [38,39]. The studies on 5-dAza effect on variety of cancer cells suggest that there is no one common mechanism in which 5-dAza induces cell death and the toxic effect of this compound is presumably related to the global and cell-specific dysregulation of the metabolism and signaling pathways [7,9-13].

Assuming this, it might be expected that 5-dAza-based therapy in combination with other selective drug enhancing the cell-specific action of the demethylating agent would be a promising therapy for treatment of different cancers. Actually, the latest clinical trials have brought the results confirming that the combination of Decitabine (5-dAza) with other agents has given promising results in AML disease therapy $[40,41]$. Moreover, recent trials with Decitabine in combination with valproic acid have shown the anti-cancer effect in NSCLC-diagnosed patients [30].

In this paper, we provide evidence that the toxic effect of 5-dAza in lung cancer cells basically relies on the alteration of mitochondrial function via overexpression of proteins involved in TCA and OXPHOS. We believe that results presented here shed more light on the mechanism of the action of the demethylating agent - 5-dAza, in lung cancer cells.

\section{Acknowledgements}

This research was supported by Polish Ministry of Science and Higher Education Grant, \#N N401 376139 and by Research Grant of Institute of Experimental Biology of Wroclaw University.

\section{References}

1. Herman JG, Baylin SB (2003) Gene silencing in cancer in association with promoter hypermethylation. N Engl J Med 349: 2042-2054.

2. Esteller M (2005) Aberrant DNA methylation as a cancer-inducing mechanism Annu Rev Pharmacol Toxicol 45: 629-656.

3. Christman JK (2002) 5-Azacytidine and 5-aza-2'-deoxycytidine as inhibitors of DNA methylation: mechanistic studies and their implications for cancer therapy. Oncogene 21: 5483-5495.

4. Fenaux P Mufti GJ, Hellström-Lindberg E, Santini V Gattermann N, et al. (2010) Azacitidine prolongs overall survival compared with conventional care regimens in elderly patients with low bone marrow blast count acute myeloid leukemia. J Clin Oncol 28: 562-569.

5. Bender CM, Pao MM, Jones PA (1998) Inhibition of DNA methylation by 5-aza2'-deoxycytidine suppresses the growth of human tumor cell lines. Cancer Res 58: 95-101.

6. Daskalakis M, Nguyen TT, Nguyen C, Guldberg P, Köhler G, et al. (2002) Demethylation of a hypermethylated P15/INK4B gene in patients with myelodysplastic syndrome by 5-Aza-2'-deoxycytidine (decitabine) treatment. Blood 100: 2957-2964.

7. Schmelz K, Wagner M, Dörken B, Tamm I (2005) 5-Aza-2'-deoxycytidine induces p21WAF expression by demethylation of p73 leading to p53independent apoptosis in myeloid leukemia. Int J Cancer 114: 683-695.

8. Herman JG, Jen J, Merlo A, Baylin SB (1996) Hypermethylation-associated inactivation indicates a tumor suppressor role for p15INK4B. Cancer Res 56 722-727.

9. Gomyo Y, Sasaki J, Branch C, Roth JA, Mukhopadhyay T (2004) 5-aza-2'deoxycytidine upregulates caspase- 9 expression cooperating with p53-induced apoptosis in human lung cancer cells. Oncogene 23: 6779-6787.

10. Liu X, Wang X, Zhang J, Lam EK, Shin VY, et al. (2010) Warburg effect revisited: an epigenetic link between glycolysis and gastric carcinogenesis. Oncogene 29: 442-450.

11. Li H, Wang J, Xu H, Xing R, Pan Y, et al. (2013) Decreased fructose-1,6bisphosphatase-2 expression promotes glycolysis and growth in gastric cancer cells. Mol Cancer 12: 110.

12. Dong C, Yuan T, Wu Y, Wang Y, Fan TW, et al. (2013) Loss of FBP1 by Snail-mediated repression provides metabolic advantages in basal-like breast cancer. Cancer Cell 23: 316-331.

13. Chen M, Zhang J, Li N, Qian Z, Zhu M, et al. (2011) Promoter hypermethylation mediated downregulation of FBP1 in human hepatocellular carcinoma and colon cancer. PLoS One 6: e25564.

14. Freeman AE, Hoffman RM (1986) In vivo-like growth of human tumors in vitro. Proc Natl Acad Sci U S A 83: 2694-2698.

15. Gilbert SF, Migeon BR (1975) D-valine as a selective agent for normal human and rodent epithelial cells in culture. Cell 5: 11-17.

16. Johansson $L$ (2004) Histopathologic classification of lung cancer: Relevance of cytokeratin and TTF-1 immunophenotyping. Ann Diagn Pathol 8: 259-267.

17. Pfaffl MW (2001) A new mathematical model for relative quantification in realtime RT-PCR. Nucleic Acids Res 29: e45.

18. Kowalski W, Nocon D, Gamian A, Kołodziej J, Rakus D (2012) Association 
Citation: Sok AJ, Gizak A, Mamczur P, Piotrowska A, Knapik A, et al. (2014) Demethylation with 5-Aza-2'-deoxycytidine Affects Oxidative Metabolism in Human and Mouse Non-small Cell Lung Cancer Cells. J Cancer Sci Ther 6: 036-044. doi:10.4172/1948-5956.1000246

of C-terminal region of phosphoglycerate mutase with glycolytic complex regulates energy production in cancer cells. J Cell Physiol 227: 2613-2621.

19. Wiśniewski JR, Zielinska DF, Mann M (2011) Comparison of ultrafiltration units for proteomic and $\mathrm{N}$-glycoproteomic analysis by the filter-aided sample preparation method. Anal Biochem 410: 307-309.

20. Wiśniewski JR, Mann M (2012) Consecutive proteolytic digestion in an enzyme reactor increases depth of proteomic and phosphoproteomic analysis. Anal Chem 84: 2631-2637.

21. Wiśniewski JR, Zougman A, Mann M (2009) Combination of FASP and StageTip-based fractionation allows in-depth analysis of the hippocampal membrane proteome. J Proteome Res 8: 5674-5678.

22. Cox J, Mann M (2008) MaxQuant enables high peptide identification rates, individualized p.p.b.-range mass accuracies and proteome-wide protein quantification. Nat Biotechnol 26: 1367-1372.

23. Wiśniewski JR, Ostasiewicz P, Duś K, Zielińska DF, Gnad F, et al. (2012) Extensive quantitative remodeling of the proteome between normal colon tissue and adenocarcinoma. Mol Syst Biol 8: 611.

24. Luber CA, Cox J, Lauterbach H, Fancke B, Selbach M, et al. (2010) Quantitative proteomics reveals subset-specific viral recognition in dendritic cells. Immunity 32: $279-289$

25. Okun JG, Lummen P, Brandt U (1999) Three classes of inhibitors share a common binding domain in mitochondrial complex I (NADH:ubiquinone oxidoreductase). J Biol Chem 274: 2625-2630.

26. Chen Q, Vazquez EJ, Moghaddas S, Hoppel CL, Lesnefsky EJ (2003) Production of reactive oxygen species by mitochondria: central role of complex III. J Biol Chem 278: 36027-36031.

27. Kantarjian H, Oki Y, Garcia-Manero G, Huang X, O'Brien S, et al. (2007) Results of a randomized study of 3 schedules of low-dose decitabine in higherrisk myelodysplastic syndrome and chronic myelomonocytic leukemia. Blood 109: 52-57.

28. Ritchie EK, Feldman EJ, Christos PJ, Rohan SD, Lagassa CB, et al. (2013) Decitabine in patients with newly diagnosed and relapsed acute myeloid leukemia. Leuk Lymphoma 54: 2003-2007.

29. Momparler RL, Bouffard DY, Momparler LF, Dionne J, Belanger K, et al. (1997) Pilot phase I-II study on 5-aza-2'-deoxycytidine (Decitabine) in patients with metastatic lung cancer. Anticancer Drugs 8: 358-368.

30. Chu BF, Karpenko MJ, Liu Z, Aimiuwu J, Villalona-Calero MA, et al. (2013) Phase I study of 5-aza-2'-deoxycytidine in combination with valproic acid in non-small-cell lung cancer. Cancer Chemother Pharmacol 71: 115-121.
31. Palii SS, Emburgh BOV, Sankpal UT, Brown KD, Robertson KD (2008) DNA methylation inhibitor 5-Aza-2'-deoxycytidine induces reversible genome-wide DNA damage that is distinctly influenced by DNA methyltransferases 1 and 3B. Mol Cell Biol 28: 752-771.

32. Gonzalez-Zulueta M, Bender CM, Yang AS, Nguyen T, Beart RW, et al. (1995) Methylation of the 5' CpG island of the p16-CDKN2 tumor suppressor gene in normal and transformed human tissues correlates with gene silencing. Cancer Res 55: 4531-4535.

33. Yoshiura K, Kanai Y, Ochiai A, Shimoyama Y, Sugimura T, et al. (1995) Silencing of the E-cadherin invasion-suppressor gene by $\mathrm{CpG}$ methylation in human carcinomas. Proc Natl Acad Sci U S A 92: 7416-7419.

34. Herman JG, Umar A, Polyak K, Graff JR, Ahuja N, et al. (1998) Incidence and functional consequences of hMLH1 promoter hypermethylation in colorecta carcinoma. Proc Natl Acad Sci U S A 95: 6870-6875.

35. Karpf AR, Moore BC, Ririe TO, Jones DA (2001) Activation of the p53 DNA damage response pathway after inhibition of DNA methyltransferase by 5-aza2'-deoxycytidine. Mol Pharmacol 59: 751-757.

36. Zhu WG, Hileman T, Ke Y, Wang P, Lu S, et al. (2004) 5-aza-2'-deoxycytidine activates the p53/p21Waf1/Cip1 pathway to inhibit cell proliferation. J Bio Chem 279: 15161-15166

37. Schomburg I, Chang A, Placzek S, Sophngen C, Rother M, et al. (2013) BRENDA in 2013: integrated reactions, kinetic data, enzyme function data, improved disease classification: new options and contents in BRENDA. Nucleic Acids Res 41: D764-D772.

38. Bigl M, Jandrig B, Horn LC, Eschrich K (2008) Aberrant methylation of human $\mathrm{L}$ - and $\mathrm{M}$-fructose 1,6-bisphosphatase genes in cancer. Biochem Biophys Res Commun 377: 720-724.

39. Song W, Wang J, Yang Y, Jing N, Zhang X, et al. (2012) Rewiring drug-activated p53-regulatory network from suppressing to promoting tumorigenesis. J Mol Cell Biol 4: 197-206.

40. Faderl S, Ravandi F, Huang X, Wang X, Jabbour E, et al. (2012) Clofarabine plus low-dose cytarabine followed by clofarabine plus low-dose cytarabine alternating with decitabine in acute myeloid leukemia frontline therapy for olde patients. Cancer 118: 4471-4477.

41. Song LX, Xu L, Li X, Chang CK, Zhang Y, et al. (2012) Clinical outcome of treatment with a combined regimen of decitabine and aclacinomycin/cytarabine for patients with refractory acute myeloid leukemia. Ann Hematol 91: 18791886. 